\title{
Pensar la educación con Guillermo Hoyos-Vásquez.
}

\section{Thinking about Education with Guillermo Hoyos-Vásquez \\ Penser l'éducation avec Guillermo Hoyos-Vásquez \\ Pensar a educação com Guillermo Hoyos-Vásquez}

Fecha de recepción: 3 DE NOVIEMBRE DE 2013/ Fecha de aceptación: 10 DE ENERO DE 2014

Encuentre este artículo en http://magisinvestigacioneducacion.javeriana.edu.co/

doi:10.11144/Javeriana.M6-13.PEGH

Escrito por Miquel Martínez-Martín UNIVERSIDAD DE BARCELONA BARCELONA, España miquelmartinez@ub.edu

Patricio Enrique Carreño-Rojas Universidad de BARCELona Barcelona, España pcarrenorojas@gmail.com

En nuestra colaboración pretendemos ofrecer una síntesis del pensamiento de Guillermo Hoyos-Vásquez sobre la educación, los valores y la ciudadanía. A mediados de la década del noventa, con ocasión de un seminario en Bogotá en el que participamos algunos miembros del grupo de investigación, tuvimos nuestra primera conversación'. Recordamos gratamente su acogida y su claridad conceptual. Su discurso era propio de un educador y también de un político. De un buen educador y de un político comprometido por el bien común y el fortalecimiento de

1 El seminario se realizó en el marco de convenio de colaboración entre nuestro grupo de investigación en Educación en Valores y Desarrollo Moral (GREM) y la Organización de Estados Iberoamericanos (OEI). En él participamos María Rosa Buxarrais y Miquel Martínez, de la Universidad de Barcelona. la democracia. Una persona capaz de movilizar con la palabra y con su ejemplo en un contexto frágil y escaso en confianza activa.

A partir de ese encuentro, a lo largo de los últimos veinte años hemos coincidido en seminarios y reuniones y hemos publicado en diferentes revistas y editoriales sobre educación en valores, educación para la ciudadanía y la profundización en la democracia. Juntos dirigimos la colección Educación en Valores que, por iniciativa de la OEI, publicó la editorial Octaedro, de Barcelona, en doce volúmenes que reunieron a más de treinta autores especializados de diferentes países de Iberoamérica y dirigida a educadores, maestros y profesionales de la educación.

Guillermo Hoyos-Vásquez colaboró con nuestra Universidad como profesor invitado en los cursos de

Para citar este artículo / To cite this article / Pour citer cet article / Para citar este artigo Martínez-Martín, M., \& Carreño-Rojas, P. E. (2014). Pensar la educación con Guillermo Hoyos-Vásquez. magis, Revista Internacional de Investigación en Educación, 6 (13), 153-160. 
posgrado y maestría en Educación en Valores, en el programa de doctorado en Educación y Democracia y en el programa de formación continua del profesorado de nuestra ciudad "Barcelona, aula de ciudadanía".

La aportación de Guillermo Hoyos-Vásquez en la sensibilización del profesorado en torno a la necesidad de la formación en valores es la más notable de las que han tenido lugar en el conjunto de Iberoamérica los últimos años. En alguno de los textos afirmaba que:

[...] en tiempos de globalización neoliberal, cuando la democracia liberal formal se convierte en estrategia de dominación, la formación en valores tiene que significar, ante todo, deconstrucción del fetiche del neoliberalismo y reconstrucción del auténtico sentido de participación democrática en clave cosmopolita con base en ciudadanas y ciudadanos libres e iguales (Hoyos-Vásquez, 2006, p. 10).

Nos unió en nuestro trabajo el convencimiento de que la educación, como derecho social fundamental, es el recurso privilegiado de legitimidad del Estado de Derecho democrático, por lo que se constituye en el lugar clave para la profundización en las democracias contemporáneas.

La educación de la ciudadanía en sociedades plurales como las nuestras debe responder, de manera coherente, a una concepción de educación que pone como objetivo primordial la formación de ciudadanas y ciudadanos protagonistas en un mundo globalizado, que quieran y estén en condiciones de contribuir a que seamos todos más libres y más iguales. Por ello, la educación en nuestro siglo es substantiva y, en cierto modo, utópica y la tarea del educador, del maestro y del profesorado es, de alguna manera, una tarea de formación, de creación y también de resistencia.

Con su habitual discurso profundo y meridiano, Guillermo Hoyos-Vásquez destaca la forma como construimos los valores, el alcance y la potencia de los sentimientos morales en tal construcción y el valor de la competencia comunicacional. Coincidimos con él en la relevancia de lo próximo y de la construcción de lo que pretende tener valor universal o ser omnicomprensivamente algo estimable y valioso. Los educadores sabemos que en lo local —el aula, la escuela, la pequeña comunidad de jóvenes o de personas adultas - trabajamos con una realidad más próxima e inmediata y que solo desde el reconocimiento de lo que nos es próximo es posible avanzar hacia lo común y en contexto global. Somos raíces y alas, decía Octavio Paz. Somos sentimiento, voluntad y razón, decía Xavier Zubiri. Somos aprendices de ciudadanía en lo local para construir ciudadanía cosmopolita. Una buena tarea educadora debe permitir entender el mundo a los que aprenden y debe contribuir a formar personas capaces de transformarlo en un lugar más justo y más libre. Con sus trabajos, Guillermo nos aporta herramientas conceptuales y reflexiones sobre la educación, la ciudadanía y el fortalecimiento de la democracia. De ellas hemos escogido las que nos parecen más significativas para pensar hoy la educación en valores democráticos.

En los párrafos que siguen pretendemos recoger algunas reflexiones esenciales que ha planteado Guillermo Hoyos-Vásquez en su preocupación permanente por una fundamentación y el desarrollo de una educación para la ciudadanía y los valores en su carácter dialógico y relacional.

El pensamiento de Guillermo Hoyos-Vásquez ha puesto de relieve que la educación actual, en su proceso de adaptación y transformación, debe aportar al fortalecimiento de una sociedad democrática. Hoyos nos advierte que las democracias débiles que han vivido (y siguen viviendo) América Latina y Europa han debilitado la capacidad de diálogo y acuerdo. En este contexto, insiste en que la manera de fortalecer las democracias es asumir esta debilidad y convertirla en el vehículo imprescindible para construir sociedades democráticas sólidas. Esto supone un posicionamiento filosófico y pedagógico de apostar por una educación ciudadana en valores tales como la participación y el pluralismo.

\section{La educación en valores como punto de partida}

El proceso de consolidación y fortalecimiento de las sociedades democráticas tiene como escenario privilegiado el ámbito educativo. Hoyos-Vásquez entiende que la educación, en sí misma, es una educación en valores. No se entiende una educación sin valores y, a su vez, la formación en valores que no se inscriba en un contexto educativo, sea en la educación formal o no formal. Los procesos educativos deben luchar por un nuevo ethos cultural que permita sobreponerse al legado de la modernidad: un problema a la educación en valores que no es fácil de abordar. Por un lado, hemos sido testigos del influjo del positivismo normativo en los procesos educativos que pretenden seguir enseñando e inculcando reglas y, en el otro extremo, en reacción al positivismo normativo, surge un escepticismo valorativo propio de la postmodernidad, que opta por no interferir en la formación del "otro", cuya argumentación es la defensa y el cuidado de la autonomía del sujeto (Hoyos-Vásquez, 1995, p. 66). Sin embargo, este escepticismo valórico tendrá una primera consecuencia: un marcado individualismo que huye de cualquier tipo de ideología dogmática. En lugar de una ideología dogmática y una escépticarelativista, la educación en valores tiene el gran desafío de posicionar una educación para la ciudadanía libre 
de ideologías reduccionistas y mirar hacia el fortalecimiento de los valores democráticos, lo cual permite la democracia como una forma determinada de gobierno $y$, ante todo "un modo de con-vivir" con el "otro", como destaca Hoyos-Vásquez, con ese "rostro" vulnerable y demandante de una respuesta ética, como ya lo ha planteado Lévinas (1991). Ese "rostro" de hombres y mujeres, de países y culturas que, sobre todo en América Latina, Guillermo Hoyos-Vásquez conoció de manera cercana.

\section{El carácter relacional de los valores}

Para Guillermo Hoyos-Vásquez no vale cualquier tipo de educación y cualquier tipo de ciudadano. Plantea la educación y también la educación en valores con un carácter relacional, porque se posiciona en una compresión antropológica del ser humano como sujeto ético en relación, es decir, un sujeto que no es isla y que va construyendo y configurando la personalidad moral con los otros y desde los otros. La alteridad, para Hoyos-Vásquez, no es etérea o atemporal, fundamentada desde una determinada ontología, sino que se encuentra inscrita en el mismo lugar y espacio que habita cada ser humano. Esta habitación es la propia comunidad, el entorno en donde se construye a sí mismo y construye éticamente con los otros. El ser humano construye y se construye, porque está en una situación de aprendizaje permanente, se reconoce como un sujeto ético siempre inacabado, siempre por hacer. De alguna manera, podemos afirmar que el ser humano se construye y, al mismo tiempo, es construido en y por la sociedad. La educación es relación, al igual que los valores morales y socio-morales lo son por su carácter relacional.

Los valores son una cualidad de la vida humana, no como algo externo y añadido, sino como algo que "llevamos en el cuerpo". Además, tienen valor en su propia naturaleza y no dependen de la mera estimación subjetiva. El "valor" de los valores, como lo afirma Cortina (1997), no puede verse por criterios o medidas de calidad, puesto que ellos valen por sí mismos. Guillermo agregaría que, en efecto, valen por sí mismos, pero su razón de ser se aprecia en una relación vinculante y comprometida, que dota de sentido a las acciones y decisiones que tomamos, como sujetos, en comunidad.

Desde la perspectiva antropológica y pedagógica de Guillermo Hoyos-Vásquez, el sujeto es un sujeto ético en relación, que se autocomprende en y desde el vínculo dialógico con el otro. Para Hoyos-Vásquez, la relación comprometida no se refiere únicamente a términos individuales, sino a una construcción dialógica para la convivencia social desde el cultivo y fortalecimiento de los valores ciudadanos, "porque la responsabilidad del sujeto moral es de sí mismo y de las situaciones que lo rodean" (Hoyos-Vásquez, 1995, p. 72). Aun tras valorar la autonomía, no pontifica sobre ella y más bien previene que no es suficiente para el despliegue de los valores que permiten construir nuestra personalidad moral desde el enfoque de una educación para la ciudadanía. Frente a los peligros de una autonomía centrada de manera exclusiva en el individuo, Guillermo nos habla de la necesidad de educar en valores como justicia, equidad y solidaridad. De lo contrario, se corre el riesgo de implantar una educación moral autocentrada en las propias expectativas y necesidades; esto propicia individualismo y relativismo moral, los cuales impiden la concreción de los proyectos colectivos y la búsqueda del bien común como un valor moral en sí mismo. Tal como lo advierte Taylor, "el lado oscuro del individualismo supone centrarse en el yo, lo que aplana y estrecha a la vez nuestras vidas, las empobrece de sentido, y las hace perder interés por los demás o por la sociedad" (1994, p. 40). Caminar en la superación de este individualismo y apatía
Descripción del artículo | Article description | Artigo descrição | Description de l'article

El artículo ofrece una síntesis del pensamiento de Guillermo Hoyos-Vásquez sobre la educación, los valores y la ciudadanía en el contexto de Iberoamérica. Resalta el aporte notable de su trabajo en este campo a la sensibilización del profesorado en torno a la necesidad de la formación en valores. Destaca cuatro de sus ideas centrales: el reconocimiento del ámbito educativo como escenario privilegiado para la consolidación de sociedades democráticas; la comprensión antropológica del ser humano como sujeto ético en relación; el papel de los sentimientos morales como criterios de acción y la libertad humana como condición de moralidad. 
requiere educar para pensar reflexivamente sobre los valores morales. En este sentido, Guillermo propone acentuar la estructura fundamentalmente contextualista porque allí se constituye el sujeto moral como "responsable de... y respecto a... en el mundo de la vida, en la historicidad, en la cultura, en la sociedad civil" (Hoyos-Vásquez, 1995, p. 72). Esto protege al sujeto moral de actitudes egocéntricas narcisistas que niegan el carácter dialógico (Taylor, 1994).

\section{Sensibilidad ética: los sentimientos morales}

Una de las aportaciones que Guillermo ofrece al mundo de la educación es su reflexión sobre las actitudes participativas que se presentan en el mundo de la vida, en las experiencias individuales y colectivas y que dan cuenta de una cierta sensibilidad ética, identificada con los sentimientos morales de "resentimiento", "indignación" y "culpa" planteados por Strawson en su obra Libertad y resentimiento (1997). El objetivo es dotar a la ética de una fundamentación fenomenológica para hablar con propiedad de una "ética para ciudadanos" (Hoyos-Vásquez, 2008). El reconocimiento de los sentimientos morales nos permite valorar las capacidades humanas en el marco de la formación en los valores ciudadanos.

Los sentimientos morales -en su carácter complementario a una ética contractualista - señalan el proceso de integración y de construcción de sentido de pertenencia a la comunidad que nos es más próxima e inmediata. Los sentimientos morales nos revelan la manera como asumimos y reaccionamos ante las situaciones de marginalidad, pobreza y exclusión y que nos obliga a educar en la solidaridad, a la que Guillermo llama "solidaridad moral" (2008, p. 157).

El resentimiento aparece cuando el sujeto ha sido privado de sus derechos en cuanto ciudadano y la dignidad humana se ve desquebrajada. La relación vinculante - en tanto dota de sentido- del ciudadano frente a la sociedad queda resentida y traerá decepción y desconfianza. La consecuencia inmediata será el rompimiento del diálogo y de la participación, lo que provoca ostracismo. La apatía de los jóvenes y de la ciudadanía en general hacia la democracia representativa es un claro ejemplo de un resentimiento en la forma como está avanzando. Pedagógicamente, conviene aprovechar este sentimiento moral negativo para denunciar la privación de derechos y apostar por la profundización en métodos más potentes y participativos de ejercicio democrático. Educar para una ciudadanía activa es formar para ejercer derechos ciudadanos, denunciar su falta e implicarse para garantizarlos.

El sentimiento moral de indignación lo apreciamos cuando, como espectadores, notamos injurias, ofensas y descalificaciones de unos contra otros. Sentir indignación ante injusticias y maltratos puede ser un punto de partida para desarrollar la empatía, practicar y valorar la solidaridad, en especial con aquellos que han sido privados de los derechos más fundamentales. En términos pedagógicos, el sentimiento moral de indignación permite iniciar procesos de responsabilidad ética, al hacernos sentir parte de una misma comunidad en la que también de nosotros dependen las consecuencias de las acciones que afectan a los demás. Sentir con el otro no es solo ponerse en su lugar; es condición necesaria para la práctica de una ciudadanía activa.

El sentimiento de culpa revela nuestras propias miserias y limitaciones que la conciencia moral se encarga de mostrar en aquellas situaciones y vivencias en las que hemos agredido a otro en su dignidad. Aparece tanto ante nuestras actitudes y acciones egoístas, como ante el acto 
intencionado de omisión frente a situaciones de injusticia y desigualdad que demandan nuestra respuesta solidaria y humana cuando somos testigos de las ofensas, las injusticias y el maltrato a la dignidad de cada sujeto. Desde nuestra perspectiva pedagógica, el sentimiento de culpa, así definido, permite fomentar el sentido de responsabilidad frente a las consecuencias negativas que nuestras acciones $u$ omisiones puedan generar. Una ciudadanía activa que denuncia la falta de derechos y que incorpora el sentir con y desde los otros, como nota esencial, debe ser también un ejercicio de responsabilidad ética.

La cuestión es de qué manera, a partir de los sentimientos morales, podemos llegar a criterios y principios que nos permitan juzgar los casos particulares desde el punto de vista moral. Lo complejo es que la moral se ocupa de los sentimientos, las vivencias y las experiencias, pero se expresa inevitablemente en juicios de valor. Por tal razón, para la fenomenología de lo moral es importante desarrollar una sensibilidad moral con el fin de reconocer y vivenciar los conflictos de valor como se nos presentan a diario - dice Hoyos-Vásquez- en el mundo de la vida y en la sociedad, para que las posibles soluciones se encuentren contextualizadas.

La sensibilidad moral es objeto de educación y aprendizaje, porque no proviene de la nada en términos ahistóricos, sino de un contexto vital y social que requiere, en todo momento, un análisis hermenéutico. Es lo que Guillermo llama "un momento hermenéutico", que inicia el proceso de avanzar hacia una consistencia moral. En esta educación y este aprendizaje, Hoyos-Vásquez reclama un papel profético, en cuanto a anuncio y denuncia, sobre todo a los medios de comunicación, a los que también exige cumplir un papel de denuncia y concientización además de una función pedagógica (Hoyos-Vásquez, 1995, p. 74). Podríamos agregar una función de visualización de los valores democráticos inherentes a la libertad de expresión, el pluralismo y la inclusión social.

Guillermo insiste en que los sentimientos, las vivencias y las experiencias propias del mundo de la vida son subjetivos, pero se inscriben en un contexto que permite su generalización. En el ámbito del mundo de la vida, conviene no confundir aquello que es subjetivo con lo que es privado. Lo subjetivo puede ser generalizable; no así lo privado. En resumen, toda experiencia que conlleve sentimientos morales puede proporcionar criterios de acción para una educación en valores democráticos como la participación y el compromiso ciudadano ${ }^{2}$. De esta forma, estamos educando al sujeto

2 La educación en los valores de participación y compromiso ciudadano supone apostar por un determinado modelo de ciudadanía y de formación (Martínez, 1998) moral, quien va configurando las distintas dimensiones de la personalidad moral que podemos visualizar en las situaciones problemáticas que se le presentan ${ }^{3}$.

\section{El "puente" desde los sentimientos morales a principios universales}

Sabemos bien que las experiencias humanas, en un primer momento, son vividas, con toda la carga emocional que supone $y$, en un segundo momento, son enunciadas, mediante los juicios de valor que hacemos sobre ellas. Ahora bien, se nos presenta el dilema de cómo pasar de los sentimientos morales, visualizados en las experiencias particulares, a los necesarios principios universales. Como bien lo expresa HoyosVásquez, se trata de una especie de "transformador".

Para ello, Guillermo plantea que nos valemos de un principio metodológico de carácter inductivo y afirma que existe un puente: la libertad humana, porque, ante las demandas y obligaciones morales, solo podemos responder a ellas libre y responsablemente. Si retomamos las palabras de Hoyos-Vásquez (1995), diremos que solo si reconocemos que somos libres podemos descubrir que la moralidad tiene sentido para el ser humano.

El posicionamiento de Hoyos-Vásquez, desde una ética discursiva, propone que este puente es un principio dialogal, que replantea la ética kantiana de obrar solo según aquella máxima que se puede querer que se convierta en ley universal. El giro discursivo radica en que "en lugar de proponer a todos los demás una máxima como válida y que quiero que sea ley general, tengo que presentarles a todos los demás mi máxima con el objeto de que comprueben discursivamente su pretensión de universalidad" (1995, p. 76). El giro ético-discursivo descansa en el planteamiento de Habermas, el cual afirma que "sólo son válidas aquellas normas de acción con las que pudieran estar de acuerdo como participantes en discursos racionales todos aquellos que de alguna forma pudieran ser afectados por dichas normas" (Habermas, 1992, citado en Hoyos-Vásquez, 1995, p. 76). Nos plantea que el fundamento de toda moral es, sobre todo, la estructura de la comunicación humana que, por el solo hecho de ser humana, requiere explicación y análisis. Así, la estructura comunicativa tiene distintos momentos.

Como ya hemos comentado, el proceso comunicativo tiene un momento inicial al que Guillermo denomina "nivel hermenéutico de la comunicación" y consiste en el respeto a la diversidad cultural y otras formas de vida y de pensamiento, basado en los valores

3 Junto con José M. Puig y otros investigadores hemos profundizado en las dimensiones de la personalidad moral (Puig, 1996; Puig \& Martín, 1998; Buxarrais, Martínez, Puig \& Trilla, 1997). 
del pluralismo y de la tolerancia. El respeto no es solo una aceptación del otro, sino el "reconocimiento del otro" (Hoyos-Vásquez, 1995, p. 76). Este reconocimiento implica un sólido compromiso por un diálogo constructivo, capaz de avanzar en una sociedad más dialogante, respetuosa, responsable e interesada en la búsqueda del bien común. Hoyos-Vásquez (1995) nos recuerda que "toda moral tiene que comenzar por la comprensión del otro".

Ahora bien, el reconocimiento y la validación del diálogo tienen por vehículo el lenguaje, porque este nos permite tomar conciencia de lo que somos y hacemos, exponer nuestras ideas, ser interlocutores válidos $y$, en particular, comunicar todo lo que nos acontece, tematizar y articular nuestras experiencias cotidianas, sobre todo aquellas experiencias vitales. Pasar por alto este momento hermenéutico comporta el peligro de pasar directamente a los acuerdos, consensos y contratos, lo que anula o evita la valoración de la diversidad y los mismos disensos. En definitiva, sin el debido análisis hermenéutico, la ética comunicativa pierde su sentido y validez.

Las morales comunitaristas proponen como principio mediador a la comunidad a la que pertenecemos, con sus tradiciones, valores y virtudes, para fortalecer una moral ciudadana que fomenta la solidaridad entre todos los miembros de comunidad local más inmediata. Estamos aquí ante los planteamientos comunitaristas que privilegian un discurso ético surgido desde la misma comunidad local a la que pertenecemos. No obstante, como bien lo advierte Hoyos-Vásquez, un lenguaje que plantea solo la preocupación por "los míos" corre el peligro de un "nacionalismo" o fundamentalismo que destruye nuestra capacidad de diálogo y de escucha activa, de solidaridad y preocupación por quienes han sido excluidos y marginados, puesto que todos ellos se encuentran fuera del círculo de "los míos" y se genera una sociedad muy poco solidaria e injusta.

Desde una ética comunicativa, del momento inicial debemos pasar a un segundo momento: el acuerdo y la validación por una ética de mínimos, porque ella nos da garantía de la validación y los acuerdos de unos mínimos valores que puedan sostener y fundamentar el orden social. La aceptación de una ética de mínimos supone un compromiso con el logro de los valores ciudadanos que integra. En este ámbito, Guillermo nos remite al contrato social de John Rawls (1991), el cual propone el valor de la justicia como el valor de mínimos fundamental expresado en los dos principios siguientes: el primero, que "toda persona tiene igual derecho a un régimen plenamente suficiente de libertades básicas iguales, que sea compatible con un régimen similar de libertades para todos". El segundo, que la inequidad y desigualdad social, por un lado, "deben estar asociadas a cargos y posiciones abiertos a todos en las condiciones de una equitativa igualdad de oportunidades" y, por otro, de manera complementaria a la anterior, se "debe procurar el máximo beneficio de los miembros menos aventajados de la sociedad" (Rawls, 1991, p. 33).

Así como Hoyos-Vásquez advierte el peligro del comunitarismo, también lo advierte del liberalismo o universalismo, cuando este pretende establecer un único modo de ver la realidad, al considerar que la realidad es una sola y de carácter universal. En el liberalismo está ausente la valoración de los aportes que cada cultura, grupo social y comunidad de primera referencia ofrece para una construcción ciudadana que valora la diversidad, la tolerancia y el pluralismo.

En el esfuerzo por superar los límites del comunitarismo y del liberalismo, Rawls formula una propuesta para que sea viable lo que llama el "contrato social". Propone que, ante la diversidad de visiones omnipresentes con pretensión de universalidad, es necesario llegar a un "pluralismo razonable", consistente en un punto de acuerdo que beneficie a todos a partir de aquellos mínimos propuestos en los principios de justicia y equidad, que comienza con los bienes primarios. El pluralismo razonable hace posible intentar un consenso acerca de los principios básicos de la justicia que Rawls plantea: la igualdad de libertades y de oportunidades y la distribución equitativa de los bienes primarios. Este pluralismo razonable faculta el discurso social que pretende alcanzar un acuerdo sobre los mínimos, que para Hoyos-Vásquez (2010) son, principalmente, los derechos humanos. El pluralismo, como valor socio-moral, cobra un carácter imperativo porque, como lo dice Hoyos-Vásquez (2010), nos obliga a comprender las diferencias sociales y culturales y valorarlas más como una oportunidad que motiva la búsqueda y consolidación de los mínimos que como obstáculo para acoger, en contexto de interculturalidad, las diferencias sociales y culturales.

Este sería el sentido de una concepción política de la justicia que Guillermo valora y extiende, porque tiene la convicción de que toda praxis política no solo es de justicia, sino también de igualdad, equidad, libertad y, sobre todo, de reconocimiento de la vulnerabilidad del otro, cuyo rostro es el más excluido, marginado y empobrecido por una sociedad de "mercado y neoliberal", quien nos demanda una urgente respuesta ética.

La respuesta ética es una ética comunicativa y dialógica que permita llegar a los consensos en virtud de una sociedad civil más justa, igualitaria e inclusiva y, para ello, es condición sine qua non el giro desde una ética kantiana hacia una dialógica, en donde el lenguaje cobra una relevancia fundamental. El lenguaje humano da a conocer la competencia ciudadana que Guillermo considera primordial: "la competencia 
comunicativa". (Hoyos-Vásquez, 2008, p. 146). Por esta misma razón, cuando nos referimos a las competencias ciudadanas, estas deben ser entendidas a partir de la competencia comunicativa (Martínez y Hoyos, 2006). Por último, debido a su carácter dialógico, la competencia comunicativa es la que desde y por medio del lenguaje, en definitiva, nos vincula a todos los seres humanos.

\section{A modo de consideración final}

Hemos seleccionado algunas de las ideas centrales del pensamiento de Guillermo Hoyos-Vásquez en función de su especial relación con la educación en valores democráticos y para una ciudadanía activa. Queremos finalizar estas páginas de reconocimiento y homenaje con una mención especial a tres ideas que nos parecen esenciales y que todo educador debiera tener presentes en su tarea. La primera, que reconocer el mundo de lo próximo y aprender a vivir y a comprender nuestros sentimientos es una condición necesaria para iniciar procesos de educación y aprendizaje que permitan crecer en sociedades plurales y globalizadas como las nuestras. La segunda, que esta condición no es suficiente, puesto que se requiere también aprender a dialogar con calidad argumentativa y rigor sobre lo propio y lo ajeno con voluntad de avanzar en los desacuerdos y buscar acuerdos, para encaminarse hacia una sociedad democrática más justa, más solidaria y más equitativa.

Y la tercera, que no bastan la deliberación, la argumentación racional, la igualdad ni la libertad como valores principales de una educación y ciudadanía colaborativa (Martínez, 2010). El valor ético de la ciudadanía es la responsabilidad. Una responsabilidad entendida en el sentido peculiar de Lévinas (1991) y que, como señala Carlos Cullen (2009), se constituye como previa a toda comprensión de la justicia como legalidad o como equidad. Es la interpelación del otro en cuanto "otro" la que nos constituye en sujetos responsables.

La educación para una ciudadanía activa comporta educar en valores democráticos, desarrollo moral y desarrollo de la competencia comunicativa - competencia ciudadana por excelencia-, aprendizajes para entender nuestro mundo y la participación en una ciudadanía colaborativa (Martínez, 2011), pero, además, debe propiciar una ciudadanía reflexiva y responsable por saberse y sentirse interpelados por el otro: una ciudadanía ética (Cullen, 2012).

Sobre estos temas conversamos con Carlos Cullen y Guillermo HoyosVásquez en nuestro último encuentro en Bogotá, en noviembre de 2012, dos meses antes de que nuestro amigo Guillermo nos dejara.

\section{Sobre los autores}

Miquel Martínez-Martín es doctor en Filosofía y Ciencias de la Educación, catedrático de Teoría de la Educación y miembro del grupo de investigación en Educación Moral (GREM) de la Universidad de Barcelona.

Patricio Enrique Carreño-Rojas es magíster en Gestión Educacional y magíster en Educación para la Ciudadanía y Valores, Universidad de Barcelona. Miembro colaborador del grupo de investigación GREM, Universidad de Barcelona.

\section{Referencias}

Buxarrais, M. R., Martínez, M., Puig, J. M. \& Trilla, J. (1997). La educación moral en primaria y en secundaria. Madrid: Biblioteca del Normalista. 
Cortina, A. (1997). Ciudadanos del mundo. Hacia una teoría de la ciudadanía. Madrid: Alianza.

Cullen, C. (2007). El malestar en la ciudadanía. Buenos Aires: La Crujía.

Cullen, C. (2009). Entrañas éticas de la identidad docente. Buenos Aires: La Crujía.

Habermas, J. (1985). Conciencia moral y acción comunicativa. Barcelona: Península.

Habermas, J. (1988). Teoría de la acción comunicativa. Madrid: Taurus.

Hoyos-Vásquez, G. (1995). Ética comunicativa y educación para la democracia. Revista Iberoamericana de Educación, 7, 65-91.

Hoyos-Vásquez, G. (2001). La filosofía política de Jürgen Habermas. Ideas y valores, 116, 132-144.

Hoyos-Vásquez, G. (2003). Ética y educación para una ciudadanía democrática. En Departamento Administrativo de Bienestar Social del Distrito (ed.). Camino hacia nuevas ciudadanías. Bogotá: Pontificia Universidad Javeriana.

Hoyos-Vásquez, G. (2008) (ed.). Filosofía de la educación No. 29. Enciclopedia Iberoamericana de Filosofía. Madrid: Trotta.

Hoyos-Vásquez, G. (2010). Ética, interculturalidad y pluralismo. En B. Toro \& A. Tallone (coords.). Educación, valores y ciudadanía. Madrid: OEl.

Hoyos-Vásquez, G. (2012). Ensayos para una teoría discursiva de la educación. Bogotá: Magisterio.

Hoyos-Vásquez, G. \& Guillen, G. (1997). La teoría de la acción comunicativa como nuevo paradigma de investigación en las ciencias sociales: las ciencias de la educación. Bogotá: ASCUN.

Hoyos-Vásquez, G. \& Martínez, M. (2004). (coords.) ¿Qué significa educar en valores hoy? Barcelona: Octaedro.

Hoyos-Vásquez, G. \& Ruiz, A. (comps.). (2008). Ciudadanías en formación. Bogotá: Magisterio.

Lévinas, E. (1991). Ética e infinito. Madrid: Visor.

Martínez, M. (1998). El contrato moral del profesorado. Condiciones para una nueva escuela. Bilbao: Desclée.

Martínez, M. (2001). Compromiso moral del profesorado. Condiciones para un proyecto de educar en la ciudadanía. En A. Cortina \& J. Conill (eds.), Educar en la ciudadanía. Valencia: Editorial.

Martínez, M. (2010). Educación y ciudadanía en sociedades democráticas: hacia una ciudadanía colaborativa. En B. Toro \& A. Tallone (coords.). Educación, valores y ciudadanía. Madrid: OEl.

Martínez, M. \& Hoyos-Vásquez, G. (coords.). (2006). La formación en valores en sociedades democráticas. Barcelona: Octaedro.

Puig, J. M. (1996). La construcción de la personalidad moral. Barcelona: Paidós.

Puig, J. M. \& Martín, X. (1998). La educación moral en la escuela: teoría y práctica. Barcelona: Edebé.

Rawls, J. (1978). Teoría de la justicia. México: Fondo de Cultura Económica.

Taylor, C. (1994). La ética de la autenticidad. Barcelona: Paidós. 